\title{
Öffentlich-private Partnerschaft im Wassersektor des Nigers : Kartographie einer Reform
}

\section{Mahaman Tidjani Alou}

\section{(2) OpenEdition}

\section{Journals}

Electronic version

URL: http://journals.openedition.org/sjep/484

DOI: $10.4000 /$ sjep.484

ISSN: 1663-9677

\section{Publisher}

Institut de hautes études internationales et du développement

\section{Printed version}

Date of publication: 1 octobre 2005

Number of pages: 165-181

ISBN: 2-88247-059-2

ISSN: 1660-5926

Electronic reference

Mahaman Tidjani Alou, « Öffentlich-private Partnerschaft im Wassersektor des Nigers : Kartographie einer Reform », Schweizerisches Jahrbuch für Entwicklungspolitik [Online], 24-2 | 2005, Online erschienen am: 08 Juni 2010, abgerufen am 08 September 2020. URL : http://journals.openedition.org/sjep/484 ; DOI : https://doi.org/10.4000/sjep.484 


\title{
Öffentlich-private Partnerschaft im Wasser- sektor des Nigers: Kartographie einer Reform
}

\author{
Mahaman Tidjani Alou*
}

D

ie Umwälzungen der vergangenen Jahre im Wassersektor der Republik Niger $^{1}$ bieten ein ideales Umfeld für die Analyse der öffentlich-privaten Partnerschaft (ÖPP), wie sie für die meisten westafrikanischen Länder typisch sind. Im Falle des Nigers sind diese Partnerschaften vor dem Hintergrund der wirtschaftspolitischen Reformbemühungen zu betrachten, die seit 1995 von den sich aufeinander folgenden Regierungen lanciert wurden und die sich unter anderem durch den Beginn der Privatisierung von Staatsbetrieben auszeichneten ${ }^{2}$. Diese Reformen, die auch den Wassersektor betreffen, beschränken sich indes nicht auf die öffentlichen Unternehmen, sondern umfassen auch die dörfliche Wasserversorgung, deren Bewirtschaftungsgrundsätze damit einen tief greifenden Wandel erlebten. Diese Umwälzungen, die sich konsequent an liberalen Grundsätzen orientieren, bieten ein ideales Terrain für das Studium der ÖPP im Wassersektor des Nigers.

Im Folgenden wird die Problematik ausschliesslich aus institutioneller Sicht erörtert $^{3}$, häufig aufgeworfene ökonomische Fragestellungen, welche sich mit den wirtschaftlichen und finanziellen Auswirkungen der Privatisierung öffentlicher Unternehmen befassen, werden dabei ausgeklammert ${ }^{4}$. In erster Linie sollen die Veränderungen verdeutlicht werden, die im Zuge der verschiedenen strukturellen Reformen der vergangenen zehn Jahre (1995-2005) im Bereich der Wasserversorgung im Niger stattgefunden haben ${ }^{5}$. Im Zentrum des Interesses steht namentlich die veränderte Rolle des Staates bei der Wasserbewirtschaftung angesichts der zunehmenden Präsenz nichtstaatlicher Akteure im Wasserbereich. War der

* Politologe, Dozent und Forscher an der Université Abdou Moumouni, Forscher am Laboratoire d'études et de recherches sur les dynamiques sociales et le développement local (LASDEL).

1 Die Republik Niger liegt in der afrikanischen Sahel-Region und bedeckt eine Fläche von 1'267'000 $\mathrm{km}^{2}$. Politisch ist das Land in 8 Regionen, 36 Departemente und 265 Gemeinden gegliedert. Auf Grund der letzten Volkszählung (2001) wird die Einwohnerzahl auf über 11 Millionen Menschen geschätzt. Angesichts seiner umfangreichen Oberflächengewässer und Grundwasservorkommen verfügt das Land über ein bedeutendes Wassernutzungspotenzial. Siehe dazu Ministère de l'hydraulique et de l'environnement, Politiques et stratégies pour l'eau et l'assainissement. De l'eau pour un développement durable, Niamey, Mai 2001.

2 S. Sidibé, La réforme du secteur parapublic au Niger, Niamey, Nouvelle Imprimerie du Niger (ohne Datum); Ministère des finances et du plan, Assemblée générale des entreprises publiques et parapubliques, rapport général, März 1996.

3 C. Ménard, „Enjeux d'eau: la dimension institutionnelle“, Revue Tiers Monde, Nr. 166, April-Juni 2001, S. 261-274 ; B. Freund, B. Lootvoet, „Où le partenariat public-privé devient l'instrument privilégié du développement économique et local. L'exemple de Durban, Afrique du Sud“, Revue Tiers Monde, Nr. 181, Januar-März 2005.

4 Siehe beispielsweise P. Plane, „La privatisation de l'électricité en Côte d'Ivoire: évaluation et interprétation des premiers résultats“, Revue Tiers Monde, Nr. 152, Oktober-Dezember 1997.

51995 hat der Niger mit der Unterzeichnung einer Absichtserklärung den Dialog mit dem IWF wieder aufgenommen. 
Staat auf diesem Gebiet während langer Zeit vorherrschend, muss er heute in allen seinen Interventionen den Dialog und die Zusammenarbeit mit anderen Akteuren suchen, die ebenfalls an den neuen Bewirtschaftungsformen im Wassersektor beteiligt sind. Der vorliegende Beitrag will die wesentlichen Elemente dieser neu entstandenen Struktur und die damit verbundene institutionelle Dynamik rund um die Wasserressourcen beschreiben und die neue Rolle des Staates erläutern, der bis anhin die Entwicklung des Sektors alleine bestimmte. Von Interesse sind dabei auch die neuen nichtstaatlichen Akteure (Verbände, Unternehmen), die auf der Bildfläche erschienen sind und Aufgaben wahrnehmen, aus denen sich der Staat zurückgezogen hat.

Damit wird die Problematik der staatlichen Hoheit in Afrika direkt angesprochen. Im Blickpunkt stehen indes in erster Linie die öffentlichen Politiken ${ }^{6}$. Anstatt jedoch diese Politiken zu beurteilen oder gar ihre Resultate zu messen, soll der Versuch unternommen werden, ihre Akteure und die von ihnen mobilisierten Ressourcen sowie die Interaktionen und neu entstandenen wechselseitigen Beziehungen im Wassersektor zu identifizieren und ihr Handeln nachzuvollziehen.

Unter diesem Ansatz betrachtet, weist die ÖPP - nicht auf globaler Ebene, sondern in vom Staat klar abgegrenzten Tätigkeitsfeldern wie beispielsweise dem Wassersektor - eine äusserst interessante Erscheinungsform auf ${ }^{7}$. Unter diesem Blickwinkel wird sowohl die urbane als auch die dörfliche Wasserversorgung untersucht, die ebenfalls von bedeutenden Umwälzungen geprägt ist.

Als Quellen dienten neben verfügbaren offiziellen Dokumenten ${ }^{8}$ auch Medienberichte $^{9}$ sowie Angaben, die im Rahmen von Gesprächen mit verschiedenen Verantwortlichen des nigerischen Wassersektors gemacht wurden ${ }^{10}$. Für eine vertiefte Betrachtung der effektiven Funktionsweise dieser Institutionen sowie ihrer Bedeutung wäre indes eine detailliertere Analyse der angestrebten politischen Zielsetzungen erforderlich gewesen. In diesem Sinne erhebt der vorliegende Beitrag keinen Anspruch auf Vollständigkeit.

Die Untersuchungen konzentrieren sich auf drei Aspekte, die eng miteinander verflochten sind: In einem ersten Schritt wird die ÖPP als Ausdruck der Reformen im nigrischen Wassersektor erörtert. Dabei werden der Kontext, in dem die Reformen stattgefunden haben, und die Argumente, die zur Legitimierung der Reformbemühungen vorgebracht wurden, gewürdigt. Danach werden die wesentlichen Elemente der Veränderungen des institutionellen Umfelds beschrieben, die im Zuge der neuesten Reformen mit der Errichtung der ÖPP stattgefunden haben, und schliesslich sollen die Konsequenzen des partnerschaftlichen Ansatzes im neu entstandenen Umfeld aufgezeigt werden.

6 B. Jobert, P. Muller, L'Etat en action. Politiques publiques et corporations, Paris, Presses Universitaires de France, 1987.

C. Ménard, op. cit.

Gesetze, Verordnungen, Dekrete, Entscheidungen.

9 Es wurden sämtliche Artikel und Interviews gesichtet, die zwischen 1993 und 2001 in der Presse erschienen sind.

10 Der Dank des Autors gilt folgenden Personen, die sich zu einem Gespräch bereit erklärt haben : Zibo Zakara, Leiter des Programme sectoriel eau; Yvan Kédaj, technischer Mitarbeiter beim für die Wasserbewirtschaftung zuständigen Ministerium; André Patétat, Generaldirektor der Société d'Exploitation des Eaux du Niger (SEEN), und Atto Indatou, Betriebsdirektor der Société de Patrimoine des Eaux du Niger (SPEN). Als wichtige Informationsquelle diente ferner die Website der Autorité de régulation multisectorielle $(\mathrm{ARM}),<\mathrm{www}$.arm-niger.org $>$. 
Die Reform des Wassersektors ist vorab als Antwort auf das Missmanagement durch die öffentliche Hand und als Definition eines neuen Rahmens für staatliche Interventionen zu betrachten.

\section{Allgegenwart des Staates}

Während langer Zeit war die Wasserbewirtschaftung im Niger durch die dominierende Stellung der technischen Dienste des Staates geprägt. Diese waren für die Erarbeitung und Umsetzung von Politiken in urbanen wie auch in ländlichen Gegenden zuständig. Dem staatlichen Handeln lag eine ausschliesslich technokratische Logik zu Grunde: Ausgehend von Annahmen über den Wasserbedarf der Bevölkerung orientierte sich die Errichtung von Wasserversorgungsinfrastrukturen an raumplanerischen Erwägungen, wobei die Meinung der Bevölkerung, die davon profitieren sollte, häufig nicht berücksichtigt wurde.

In urbanen Zonen wurde die Trinkwasserversorgung durch einen Staatsbetrieb sichergestellt. Die urbane Wasserversorgung war ursprünglich dem staatlichen Elektrizitätsversorgungsunternehmen (Société Anonyme pour la Fourniture d'Electricité, SAFELEC, später Société Nigérienne d'Electricité, NIGELEC) angegliedert und wurde ab 1987 mit der Gründung der Société Nationale des Eaux $(\mathrm{SNE})^{11}$ in die Eigenständigkeit übergeführt. Damals galt es, die Verwaltung des Sektors, der bis anhin über keinerlei Autonomie verfügte, aufzuwerten. In der Tat hatte der Staat dem Elektrizitätsunternehmen bei der Bewirtschaftung des Wassersektors völlig freie Hand gelassen. Unter diesen Umständen bestand natürlich keinerlei Hoffnung auf finanzielle Unterstützung beim Ausbau der Wasserversorgung angesichts der rasanten Urbanisierung. Die mit Geldern der Weltbank finanzierte Gründung der SNE erschien in diesem Kontext als zweckmässige Lösung, um den Investitionsbedarf in diesem strategisch wichtigen Sektor zu decken, der immer weniger in der Lage war, die Wasserversorgung für die Bevölkerung in den urbanen Regionen sicherzustellen.

Für die Trinkwasserversorgung in ländlichen Gebieten war das OFEDES ${ }^{12}$, ein weiteres vom Staat gegründetes öffentliches Unternehmen zuständig. Dieses verfügte über folgende exklusive Kompetenzen:

11 Als Gesetzesgrundlage für die Errichtung der SNE diente die „Ordonnance No. 87-31 du 24 septembre 1987 portant création de la Société Nationale des Eaux (SNE)“ (Verordnung über die Gründung der SNE). Der Unternehmenssitz befindet sich in Niamey. Siehe Ministère de l'hydraulique et de l'environnement, Programme hydraulique Suisse Niger, Le rôle de l'Etat dans la gestion des ressources naturelles. Recueil des textes législatifs et réglementaires, Niamey, 1999. Die SNE verfügt über Niederlassungen in allen Regionen des Landes und befindet sich ausschliesslich im Staatseigentum. Zum Zeitpunkt seiner Privatisierung zählte das Unternehmen 540 Mitarbeitende und betrieb 51 Zentren, 8 Pumpwerke, 3 Wasseraufbereitungsanlagen, 85 Reservoirs und 124 Wasserbohrungen. Die Gesamtlänge des Versorgungsnetzes betrug 1600 km. Darüber hinaus bewirtschaftete das Unternehmen 1400 öffentliche Brunnen sowie 2 Trinkwasserwerke. Siehe Le Républicain, 14.12.2000, S. 8.

12 Das OFEDES ist ein Handels- und Industrieunternehmen, das 1963 mit dem Ziel gegründet wurde, Wasserbohrungen durchzuführen und Brunnen zu errichten. Siehe auch Alternative Magazine, No. 00, 24. 2.1996 sowie die „Ordonnance No. 74-32 du 15 novembre 1974 portant création d'un établissement public chargé de l'exploitation des eaux du sous sol" (Verordnung über die Gründung eines öffentlichen Unternehmens zur Bewirtschaftung der Grundwasserressourcen). Mit dieser Verordnung wurde das Gesetz „Loi No. 61-31 du 7 mai 1963 portant création d'un établissement public chargé de 
$\square$ Bau von Brunnen durch Einsatz von Humankapital ${ }^{13}$ oder im Auftragsverhältnis ${ }^{14}$ sowie Errichtung von Wasserbohrungen in urbanen Zonen und landwirtschaftlichen Gebieten;

- Bewirtschaftung, d.h. Unterhalt und Betrieb von Brunnen und Wasserbohrungen in ländlichen und Weidegebieten und der dafür erforderlichen Infrastruktur;

๑ Errichtung von Trinkwasserversorgungsnetzen in sekundären Zentren.

Damit kontrollierte das OFEDES nicht nur die Produktion und die Verteilung von Trinkwasser in ländlichen Gebieten ${ }^{15}$, sondern auch den Unterhalt der von ihm errichteten Wasserversorgungsinfrastruktur ${ }^{16}$. Auch hier ging die Dynamik von staatlicher Seite aus: Durch den Ausbau und die Erweiterung der Infrastruktur sollte längerfristig die Wasserversorgung für die Bevölkerung in den ländlichen Regionen des Nigers gewährleistet werden.

Die gesamten 60er und 70er Jahre hindurch nahm der Staat im Wassersektor eine dominierende Stellung ein. Diese Rolle, die sich die Behörden selbst zugewiesen hatten, wurde indes in keiner Weise hinterfragt, denn sie entsprach der damaligen Auffassung von politischem Handeln: Der Staat war gewillt, alles selbst in die Hand zu nehmen. Die Auffassung, wonach der Staat ein massgebender Akteur der wirtschaftlichen und gesellschaftlichen Entwicklung sei, entsprach einem breiten Konsens. Diese Entwicklung im Wassersektor ist typisch für zahlreiche andere Bereiche, die de facto als angestammte Handlungsfelder des Staates angesehen wurden. Allerdings wurden die Grenzen der staatlichen Vormachtstellung im Wassersektor schon bald erkennbar.

\section{Schema 1: Der Wassersektor vor der Errichtung der ÖPP}

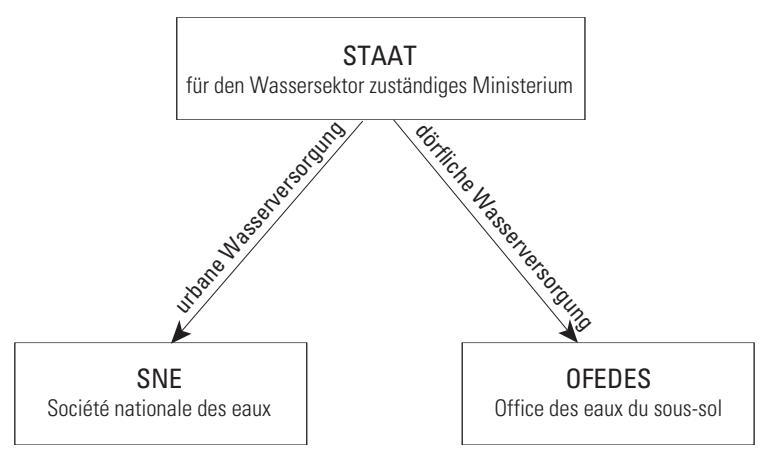

l'exploitation des eaux de sous-sol dans la République du Niger" (Gesetz über die Gründung eines öffentlichen Unternehmens zur Bewirtschaftung der Grundwasserressourcen) revidiert, welche bereits durch das Gesetz Nr. 73-14 vom 5. April 1974 geändert worden war.

13 Damit ist die Beteiligung der Bevölkerung beim Bau von Wasserversorgungsanlagen gemeint.

14 Als beauftragtes Unternehmen war das OFEDES in der Lage, mit der Durchführung von Arbeiten Erträge zu erwirtschaften.

15 Ordonnance No. 74-32 vom 15. November 1974, op. cit.

16 Siehe „Loi 94-21 du 6 septembre 1994 portant création d'un établissement public à caractère industriel et commercial dénommé Office des eaux du sous-sol (OFEDES)“ (Gesetz über die Gründung eines öffentlichen Industrie- und Handelsunternehmens namens Office des eaux du sous-sol OFEDES). In diesem Gesetz wurde die Vormachtstellung des OFEDES im Bereich der dörflichen Wasserversorgung verankert. 


\section{Legitimitätsverlust des Staates}

Die Allmacht des Staates erwies sich trotz der bedeutenden Investitionen, die sie generiert hatte, als wenig fruchtbar. Die politischen Bemühungen trieben die Entwicklung des Wassersektors nicht in befriedigendem Masse voran. Das Missmanagement griff rasch um sich und wurde zur Norm. Solange jedoch der Staat und die internationale Gemeinschaft die enormen Investitionen zu finanzieren vermochten, nach denen der Wassersektor verlangte, waren die Funktionsmängel nicht sichtbar. Erst die Haushaltskrise - verstärkt durch den abnehmenden Geldfluss aus dem Ausland, nachdem der Niger sich geweigert hatte, sein zweites Strukturanpassungsprogramm zu unterzeichnen - und die Unruhen im Vorfeld der Nationalkonferenz von 1991 führten den Kollaps herbei.

Die Krise liess die öffentliche Wasserbewirtschaftung in den Städten wie auch auf dem Land in einem neuen Licht erscheinen und machte die Grenzen des seit der Unabhängigkeit vorherrschenden Modells deutlich. Wie bereits erwähnt, waren die staatlichen Strukturen nicht in der Lage, eine effiziente Bewirtschaftung des Wassersektors sicherzustellen. Die SNE war abhängig von Finanzspritzen der öffentlichen Hand, die ihrerseits nicht in der Lage war, die Wasserrechnung der Verwaltung zu begleichen. Bei der dörflichen Wasserversorgung war vor allem die Untauglichkeit des Unterhaltssystems problematisch (gemeinschaftliche Verwaltung unter Beteiligung der Gebietskörperschaften), da sich der Staat angesichts der sinkenden Erträge aus der Uranförderung und der seit Ende der 80er Jahre spärlicher fliessenden internationalen Finanzhilfe mehr und mehr zurückzog. Bald zeigte sich, dass die Wasserversorgungsanlagen, die vom Staat in den drei Jahrzehnten seit der Unabhängigkeit errichtet worden waren, nicht nachhaltig waren. Das Missmanagement und dessen Auswirkungen auf den Unterhalt der Anlagen trugen zur desolaten Situation bei.

De facto war es also die Ende der 80er Jahre beginnende Staatskrise, welche den Ausschlag für die Veränderungen in der Wasserbewirtschaftung gab. Eingebettet in die umfassenden politischen Strukturanpassungsbemühungen führten diese Veränderungen dazu, dass zahlreiche bisher dem Staat vorbehaltene Privilegien dem Privatsektor und den dörflichen Gemeinschaften übertragen wurden. Dieser in der Staatskrise wurzelnde Befugnistransfer wurde durch die Geldgeber des Nigers weiter gefördert: Sie nutzten die strukturelle Schwäche des Staates, um ihn neu auszurichten, indem sie die Gewährung von Geldern an neue Richtlinien für die Bewirtschaftung der Wasserressourcen knüpften. Im Laufe der 90er Jahre wurde denn auch mit der Verabschiedung verschiedener Gesetze und Verordnungen ${ }^{17}$ die rechtliche Grundlage für die Errichtung eines neuen institutionellen Rahmens geschaffen.

17 Als allgemeine gesetzliche Grundlage für die Organisation des Wassersektors im Niger dient eine 1993 beschlossene und 1998 revidierte Verordnung über die Wasserbewirtschaftung (Ordonnance No. 93-014). 


\section{Politische Neuausrichtung im Wassersektor: Der Weg zur öffentlich-privaten Partnerschaft}

Anlass für die politische Neuausrichtung im Wassersektor, welche in die Errichtung einer ÖPP mündeten, waren die schwerwiegenden Missstände im Bereich der urbanen, wie auch der dörflichen Wasserversorgung. Es galt, eine Antwort zu finden auf die mangelnden Investitionen der öffentlichen Hand im Wassersektor und das konsequente Scheitern aller bisherigen politischen Massnahmen. Immer häufiger knüpften die Geldgeber ihre Investitionsbereitschaft an die Bedingung, dass Reformen durchgeführt wurden. Bei der urbanen Wasserversorgung wurde zunächst eine Umstrukturierung der SNE und in einem späteren Schritt deren Privatisierung gefordert. Im Oktober 1996 beschloss die Regierung im Anschluss an die Mission eines Weltbank-Beraters, diesen Staatsbetrieb auf die Liste der zu privatisierenden Unternehmen zu setzen ${ }^{18}$. In der dörflichen Wasserversorgung setzten sich - unterstützt durch die neue Ausrichtung der Geldgeber des Sektors - partizipative Bewirtschaftungsmodelle durch ${ }^{19}$. Überall wurde der Bau von Versorgungsanlagen an die Voraussetzung gekoppelt, dass die Bevölkerung an der Errichtung und Bewirtschaftung der Anlagen beteiligt wird und offenere Bewirtschaftungsmodi eingeführt werden (partnerschaftliche Bewirtschaftung bzw. Bewirtschaftung auf Mandatsbasis), bei denen der Staat auf seine frühere zentrale Rolle verzichtet.

Um die Neuausrichtung zu besiegeln, richtete die nigrische Regierung zwei sektoralpolitische Erklärungen an die Weltbank. Damit verpflichtete sie sich, im Bereich der Wasserversorgung Reformen durchzuführen, welche die Stellung des Staates in der Bewirtschaftung neu regeln. Auf der Ebene der urbanen Wasserversorgung zielten die Reformabsichten in verschiedenen Bereichen auf einen Kompetenztransfer vom Staat hin zu anderen Akteuren:

๖ Eigenverantwortliche Beteiligung lokaler Gemeinschaften an der Wasserbewirtschaftung durch die Übertragung gewisser Zuständigkeiten vom Staat an dezentrale lokale Körperschaften;

ـ Stärkung der Rolle des Privatsektors und der Zivilgesellschaft bei der Wasserbewirtschaftung durch die Entwicklung ihrer Kenntnisse und Fähigkeiten im Hinblick auf den Bau von Anlagen und Infrastrukturen;

$\checkmark$ Umfassende Beteiligung der Bevölkerung bei der Planung und Durchführung von Arbeiten, Verbesserung des Unterhalts der Infrastruktur, Klärung und Anerkennung der Rollen der einzelnen Partner (Staat, Körperschaften, Privatsektor, Bevölkerung) und Sicherung der Nutzungsrechte ${ }^{20}$.

Die Tendenz des Staates, sich gegenüber anderen Akteuren zu öffnen, ist auch in Bezug auf die dörfliche Wasserversorgung erkennbar. Zu den erklärten Zielen in diesem Bereich zählen unter anderem die Stärkung der Rolle des Privatsektors

18 Ordonnance No. 96-062 vom 22. Oktober 1996.

19 Siehe die entsprechende Aufstellung in A. Hassane, A. Karadji, F. Balmer (dir.), Bien gérer l'eau du Sahel. Regards sur vingt ans de coopération entre la Suisse et le Niger dans le secteur de l'hydraulique, Berne, Direction du développement et de la coopération (DDC); Niamey, Ministère de l'hydraulique et de l'environnement; Genève, IUED, 1996.

20 Siehe Ministère du plan, Ministère des ressources en eau, Lettre de politique sectorielle de l'hydraulique urbaine, Niamey, 8.3.2001, S. 2. 
und die umfassende Beteiligung der Zivilgesellschaft (Verbrauchervereinigungen) bei der Bewirtschaftung und beim Bau von Versorgungsanlagen und -infrastrukturen im Rahmen eines Mandats ${ }^{21}$.

Seit 2001 stützt sich die gesamte Wasserversorgungspolitik des Staates auf die ÖPP ab. Sie ist Bestandteil eines umfassenden Reformprozesses und verfolgt namentlich folgende Zielsetzungen:

๑ Verbesserung der technischen und finanziellen Leistungsfähigkeit des Untersektors;

๑ Gewährleistung einer effizienten Bewirtschaftung nach ökonomischen Grundsätzen;

๑ Erhöhung der Trinkwasserversorgungsdichte namentlich im Hinblick auf einkommensschwache Bevölkerungsgruppen;

$\checkmark$ mittelfristige Sicherstellung des finanziellen Gleichgewichts des Untersektors ${ }^{22}$.

Diese Politik sollte es ermöglichen, die zahlreichen Probleme zu lösen, mit denen der Staat auf dem Gebiet der Wasserversorgung konfrontiert war. Konkret mündete sie in die Errichtung einer neuen institutionellen Architektur als Grundlage für das künftige partnerschaftliche Vorgehen.

\section{Die ÖPP als Ausdruck eines neuen institutionellen Rahmens}

Die Reform des Wassersektors im Niger ist vor dem Hintergrund des Strukturanpassungsprogramms zu betrachten und beinhaltet eine Neudefinition und Neuausrichtung der Rollen der an der Wasserbewirtschaftung beteiligten Akteure. Das Ergebnis ist eine Neuordnung sämtlicher Aspekte der Wasserversorgung.

\section{Neuordnung der urbanen Wasserversorgung}

Die Reform des Wassersektors beruhte im Wesentlichen auf einer strukturellen Erneuerung. In einem ersten Schritt wurde die bislang vom Staat dominierte urbane Wasserversorgung für neue Versorgungsunternehmen zugänglich gemacht, wodurch die Privatisierungspolitik im Wassersektor nach und nach ihre heutige Gestalt annahm. Zur Unterstützung des Reformprozesses wurden begleitende Massnahmen getroffen.

\section{$\square$ Neue Versorgungsunternehmen: Entstehung eines vierpoligen Systems}

Vier Pole charakterisieren die Neuordnung der urbanen Wasserversorgung: der Staat, die Société de Patrimoine des Eaux du Niger (SPEN), die Société d'Exploitation des Eaux du Niger (SEEN) und die Autorité de régulation multisectorielle (ARM, multisektorielle Regulierungsbehörde).

\section{Der Staat}

Der Staat ist zwar nach wie vor im Wassersektor präsent, seine Kompetenzen sind indes auf klar abgegrenzte hoheitliche Aufgaben beschränkt. Die Beziehungen

21 Ibid., S. 3.

22 Siehe hierzu die im Auftrag der SEEN durchgeführte Studie Assistance du maître d'ouvrage „SPEN“ à la réalisation des bornes fontaines, rapport final, September 2004, S. 2. 
zwischen dem Staat und seinen neuen Partnern in der öffentlichen Wasserbewirtschaftung des Nigers sind in einer entsprechenden Vereinbarung geregelt ${ }^{23}$. Der Staat definiert die Grundlagen der Sektoralpolitik und der Bewirtschaftung der Wasserressourcen, schafft den erforderlichen gesetzlichen und reglementarischen Rahmen und legt die Preispolitik fest ${ }^{24}$. Darüber hinaus überträgt er seine angestammten Vorrechte einer neu gegründeten Gesellschaft. Nicht zuletzt ist er als grösster Wasserverbraucher und bedeutendster Schuldner der ehemaligen SNE verpflichtet, seine Wasserrechnung pünktlich zu begleichen und in den Behörden vermehrt für einen sparsamen Umgang mit Wasser zu sorgen.

\section{Die Société de Patrimoine des Eaux du Niger (SPEN)}

Die SPEN wurde am 14. August 2000 als staatliches Unternehmen gegründet. Im Rahmen der Reform wurden ihr auf der Grundlage eines Konzessionsvertrags die Nutzungsrechte an öffentlichen Naturgütern und Anlagen des Untersektors der urbanen und semiurbanen Wasserversorgung und die Zuständigkeiten für deren physische, betriebliche und finanzielle Bewirtschaftung übertragen. Zu den öffentlichen Naturgütern zählen die Fliessgewässer, Seen, Weiher, Quellen, Grundwasservorkommen sowie Thermal- und Mineralquellen, während die Anlagen alle von den Gemeinwesen errichteten und genutzten Wasserversorgungseinrichtungen sowie die öffentlich zugänglichen Brunnen umfas$\operatorname{sen}^{25}$. Damit wurden Güter in enormem Umfang auf die SPEN übertragen, die nunmehr für die Bewirtschaftung des staatlichen Eigentums im Bereich der urbanen und semiurbanen Wasserversorgung verantwortlich ist. Als Bewirtschafterin ist die SPEN nicht nur für die Durchführung sämtlicher Investitionen, sondern auch für die Beschaffung der dazu erforderlichen Mittel verantwortlich. Als Generalunternehmen und Bauherrin ist sie verpflichtet, für den Unterhalt und die Erneuerung der Wasserversorgungsanlagen sorgen, und nicht zuletzt muss sie die Öffentlichkeit für den sparsamen Umgang mit Wasser und für die Reinhaltung der Wasserressourcen sensibilisieren ${ }^{26}$. Die Beziehungen zwischen Staat und SPEN sind in einem Planungsvertrag geregelt, welcher dem Konzessionsvertrag zwischen den Parteien als Anhang beigefügt ist.

\section{Die Société d'Exploitation des Eaux du Niger (SEEN)}

Die SEEN wurde am 1. Juni 2001 im Rahmen der von der Regierung lancierten Privatisierungspolitik als privatrechtliches Unternehmen gegründet. Sie wurde auf Grund eines Pachtvertrags mit der Gewinnung, der Beförderung und der Verteilung von Trinkwasser in urbanen und semiurbanen Zentren beauftragt. Alle 51 bislang von der SNE bewirtschafteten Zentren wurden der SEEN übertragen ${ }^{27}$. Darüber hinaus führt die SEEN auch gewisse Arbeiten zur qualitativen Verbesserung der Wasserversorgung durch ${ }^{28}$. Die SEEN bildet den Angelpunkt

23 Siehe dazu den am 20. März 2001 unterzeichneten Konzessionsvertrag zwischen der Republik Niger und der Société de Patrimoine des eaux du Niger (SPEN).

24 „Loi 2000-12 du 14 août 2000, portant réorganisation de l'activité de production, transport et distribution de l'eau dans le sous-secteur de l'hydraulique urbaine et créant la Société de Patrimoine des Eaux du Niger (SPEN)“ (Gesetz über die Reorganisation der Trinkwassergewinnung, -verteilung und -versorgung und über die Gründung der Société de Patrimoine des Eaux du Niger (SPEN), Artikel 2.

25 Ibid., Artikel 10.

26 Seyni Salou, Generaldirektor der SPEN, Interview in Sahel Dimanche, 7.12.2001, S. 4.

27 Seither ist zu den ursprünglich 51 Zentren ein weiteres hinzugekommen. Die Verpachtung ist als dynamischer Prozess zu verstehen, da sie die Möglichkeit bietet, das bewirtschaftete Gebiet zu erweitern.

28 ARM, <www.arm-niger.org > > Eau > Le secteur de l'eau au Niger. 
der ÖPP im Niger, welche durch den halbstaatlichen Charakter der übrigen Akteure im Bereich der Wasserversorgung geprägt ist. Mit der Gründung der SEEN wurde der Wassersektor des Nigers für die Privatwirtschaft geöffnet. Mit einer $51 \%$ igen Beteiligung ist das Unternehmen Véolia, die Nachfolgerin der früheren Vivendi Water, die Hauptaktionärin der SEEN. 34 Prozent der Aktien wurden von privaten Investoren aus dem Niger und weitere 10 Prozent von den Mitarbeitenden der früheren SNE erworben. Lediglich 5 Prozent der Anteile an dem neuen Unternehmen, das von einem weltweit tätigen Versorger geführt wird, befinden sich im Besitz des Staates ${ }^{29}$. Die entstandene Partnerschaft unterscheidet sich insofern vom klassischen Modell, als sie durch ein international tätiges privates Unternehmen von Weltruf mit umfassender Erfahrung im Bereich der Wasserversorgung angeführt wird, welches der neu geschaffenen Struktur ein solides Fundament bietet. Gemäss der Leistungsvereinbarung im Anhang zum Konzessionsvertrag steht die SEEN unter der Aufsicht der SPEN, welche den Reformprozess begleitet, und einer Regulierungsbehörde.

\section{Die Autorité de régulation multisectorielle ARM (multisektorielle Regulierungsbehörde)}

Die ARM vervollständigt die institutionelle Struktur des Wassersektors. Obwohl bereits $1999^{30}$ ins Leben gerufen, nahm sie ihre Tätigkeit erst einige Zeit nach der Gründung der SPEN und der SEEN, nämlich im Jahr $2003^{31}$ auf. Die ARM ist eine unabhängige, finanziell und verwaltungstechnisch autonome juristische Person. Ihre Beschlüsse und Entscheidungen haben den Stellenwert von Amtshandlungen und können vor Gericht angefochten werden ${ }^{32}$. Die ARM ist für die Regulierung der Tätigkeiten im Wasser-, Energie-, Telekommunikations- und Transportsektor des Nigers zuständig ${ }^{33}$. Ihre Regulierungsaufgaben umfassen mehrere Aspekte:

口 Objektive, transparente und nicht diskriminierende Überwachung der Einhaltung der für die einzelnen Sektoren massgebenden Gesetze und Regelungen;

$\checkmark$ Wahrung der Interessen von Verbrauchern und Versorgern durch die Gewährleistung eines gesunden und lauteren Wettbewerbs in den Sektoren im Rahmen der geltenden gesetzlichen und reglementarischen Bestimmungen;

๖ Förderung der Entwicklung des Sektors, insbesondere durch die Gewährleistung des wirtschaftlichen und finanziellen Gleichgewichts und durch die Erhaltung der für den Fortbestand notwendigen wirtschaftlichen Rahmenbedingungen;

29 M. Laimé, „Main basse sur l'eau des villes“, Le Monde diplomatique, März 2005, S. 16-17. Siehe auch „Saga Vivendi Universal: un rêve en échec“, Le Monde: Dossiers et Documents, Nr. 320, Mai 2003.

30 „Ordonnance No. 99-044 du 26 octobre 1999 portant création, organisation et fonctionnement d'une autorité de régulation multisectorielle“ (Verordnung über die Errichtung, Organisation und Arbeitsweise einer multisektoriellen Regulierungsbehörde).

31 Im bereits erwähnten Interview erklärte Seyni Salou, der Generaldirektor der SPEN, die ARM habe ihre Tätigkeit bereits 2001 aufgenommen. Einem Bericht im Mitteilungsblatt Le Régulateur (Nr. 00, Dezember 2004, S. 5) ist jedoch zu entnehmen, dass die Mitglieder des Conseil national de régulation (Nationaler Rat für Regulierungsfragen) erst im Dezember 2003 vereidigt wurden.

32 „Ordonnance No. 99-044“, Artikel 1, Absatz 2.

33 Ibid., Artikel 2, Absatz 1. 
$\square$ Umsetzung der in den Gesetzen und Reglementen vorgesehenen Mechanismen für die Anhörung von Verbrauchern und Versorgern ${ }^{34}$.

Die ARM verfügt über eine eigene Sanktionsgewalt, die sie entweder von Amtes wegen oder aber auf Ansuchen eines Berufsverbandes, einer Verbrauchervereinigung oder interessierter natürlicher oder juristischer Personen ausübt ${ }^{35}$. Ferner steht sie den Behörden beratend zur Seite und ist verpflichtet, die Öffentlichkeit $\mathrm{zu}$ informieren, namentlich über ihr offizielles Mitteilungsblatt Le Régulateur und über ihre Website.

Sie steht unter der Leitung des Conseil national de régulation (CNR, Nationaler Rat für Regulierungsfragen). Dieser setzt sich aus einem Vorsitzenden und den Direktoren der vier Sektoren zusammen. Ihr Ansprechpartner im Wassersektor ist der Direktor des Sektors, der wie seine Amtskollegen im Rahmen einer öffentlichen Ausschreibung ernannt wird. Dieser innovative Ansatz ist bemerkenswert und dürfte der neu geschaffenen Behörde die erforderliche Unabhängigkeit verschaffen.

Mit der Errichtung der ARM sollte, laut Vorsitzender des Conseil national de régulation, an die am Privatisierungsprozess beteiligten Akteure ein deutliches Signal für die Transparenz in der Bewirtschaftung des nunmehr privatisierten Sektors ausgesendet werden ${ }^{36}$. Diese Erklärung zielt in dieselbe Richtung wie jene des Generaldirektors der SPEN : Dieser hatte die ARM als „Schiedsrichter“ zwischen den Akteuren des Wassersektors bezeichnet, der die Einhaltung der Spielregeln - lauterer Wettbewerb, Transparenz, Einhaltung von Vereinbarungen - gewährleistet und auf diese Weise für stabile Beziehungen namentlich zwischen Staat und SPEN, zwischen SPEN und SEEN sowie zwischen SEEN und Verbrauchern sorgt ${ }^{37}$.

Die Umsetzung dieses neuen institutionellen Dispositivs wird begleitet durch das so genannte „Projet sectoriel eau“, das Programm für die Weiterentwicklung des Wassersektors.

\section{$\square$ Das Projet sectoriel eau (PSE) als Begleitstruktur}

Das PSE versteht sich als Antwort auf den Investitionsbedarf im Wassersektor. Die durchgeführten Reformen waren eine Voraussetzung für den Zugang zu den Krediten, die im Rahmen der Umsetzung des Projektes gewährt wurden. Finanziert wird das Projekt durch vier Geldgeber, nämlich die Weltbank, die Agence française de développement (AFD), die Westafrikanische Entwicklungsbank (BOAD) und die chinesische Regierung. Das Projekt im Umfang von schätzungsweise 80 Millionen Euro erstreckt sich über den Zeitraum 2002-2006 und zielt unter anderem darauf ab, durch Investitionen das Versorgungsnetz der 51 von der SEEN bewirtschafteten Zentren auszubauen und damit die begonnenen Reformen in der nigrischen Wasserversorgung zu unterstützen. Darüber hinaus soll das Projekt zur Ergänzung und Klärung des gesetzlichen und reglementarischen Rahmens für die Wasserbewirtschaftung beitragen ${ }^{38}$.

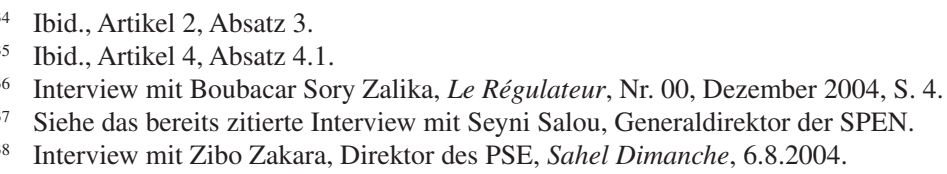


Im Bereich der urbanen Wasserversorgung sind verschiedene Massnahmen geplant:

๑ Erneuerung und Ausbau der Anlagen für die Gewinnung, Beförderung und Verteilung von Trinkwasser durch die SEEN;

ـ Planung kleinerer Ausbauvorhaben und gemeinschaftlicher Anschlüsse durch die SEEN im Rahmen der Arbeiten, die auf der Grundlage des Pachtvertrages in Auftrag gegeben werden;

$\checkmark$ Bau von öffentlichen Brunnen durch die SEEN ${ }^{39}$.

\section{Schema 2: Die ÖPP im Wassersektor des Nigers - Urbane Wasserversorgung}

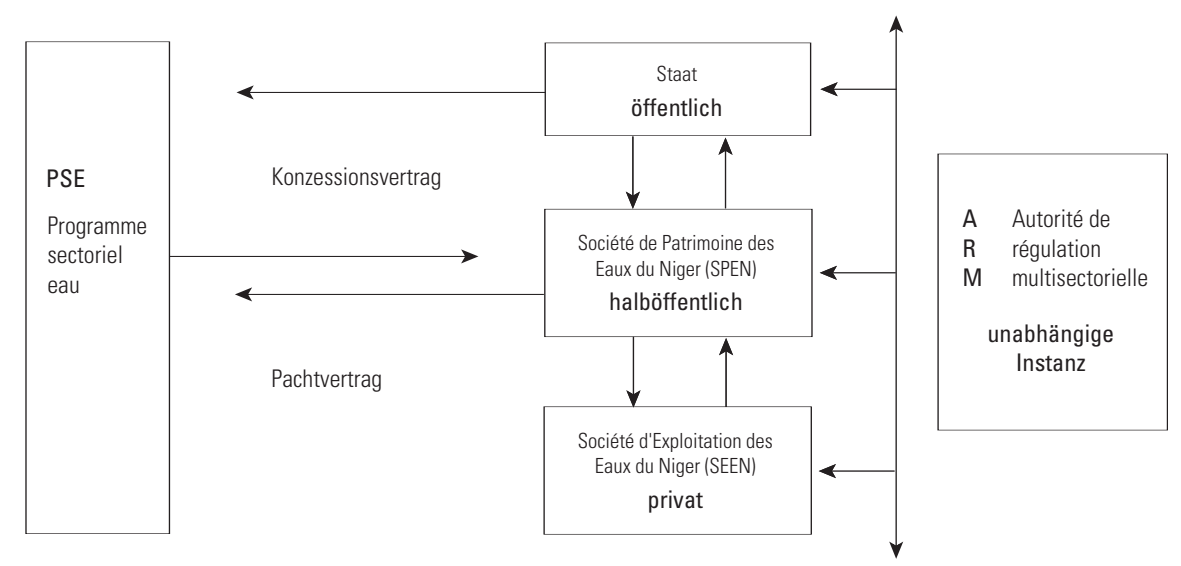

\section{Vordringen privater Akteure in die dörfliche Wasserversorgung}

Von den Veränderungen im Bereich der öffentlichen Wasserbewirtschaftung ist auch die dörfliche Wasserversorgung betroffen. Diese Bewirtschaftung wurde bislang vom Staat oder von den dörflichen Gemeinschaften sichergestellt, war aber in den meisten Fällen mangelhaft. Angesichts ihres Unvermögens, die Voraussetzungen für eine nachhaltige Bewirtschaftung der Versorgungsanlagen zu schaffen $^{40}$, wird diese immer häufiger von privaten Akteuren auf der Grundlage der so genannten „gestion déléguée“, das heisst auf Mandatsbasis, sichergestellt, welche in den geltenden Gesetzestexten als mögliche Bewirtschaftungsform ausdrücklich verankert ist ${ }^{41}$. Dabei wird die Bewirtschaftung der Wasserversorgungsanlagen gegen eine entsprechende Gebühr einem Privaten übertragen. Diese Bewirtschaftungsform ist gegenwärtig im Zunehmen begriffen, insbesondere im Bereich der kleinräumigen Wasserversorgungsnetze.

39 Siehe dazu die bereits zitierte Studie der SEEN, S. 2.

40 A. Elhadj Dagobi, J.-P. Olivier de Sardan, „La gestion communautaire sert-elle l'intérêt public? Le cas de l'hydraulique villageoise au Niger", Politique africaine, Nr. 80, 2000, S. 153-168.

41 „Ordonnance No. 93-014 du 2 mars 1993 portant régime de l'eau“ (Verordnung über die Wasserbewirtschaftung), revidiert durch das Gesetz Nr. 98-041 vom 7. Dezember 1998. 
Die Versorger lassen sich in zwei Kategorien einteilen:

$\checkmark$ individuelle Versorger; dabei handelt es sich um Handelsunternehmen oder Wasserversorgungsspezialisten, die sich auf die Bewirtschaftung kleinräumiger Versorgungsnetze spezialisiert haben;

$\checkmark$ mehrere in einem Unternehmen zusammengeschlossene Versorger, die die Wasserversorgung für mehrere Dörfer sicherstellen.

Damit entsteht in den Dörfern ein relativ gut entwickelter und strukturierter Handelssektor. War der Handel mit Wasser in ländlichen Gegenden noch bis vor kurzer Zeit quasi ein Tabu, wird er heutzutage allgemein akzeptiert ${ }^{42}$. Die Zahl der Versorger nimmt laufend zu, denn der Handel mit Wasser in den Dörfern ist äusserst lukrativ.

Vor diesem Hintergrund nimmt die öffentlich-private Partnerschaft eine besondere Form an, indem sie die Gemeinden mit einbezieht. Im Unterschied zu den urbanen und semiurbanen Zonen, wo ein einziges grosses Versorgungsunternehmen nach dem Vorbild der SEEN vorherrscht, ist der Markt von zahlreichen Verbrauchervereinigungen geprägt, die unter besonderen im Gesetz vorgesehenen Umständen die Bewirtschaftung der Wasserversorgung einem privaten Versorger übertragen. Für die Bewirtschaftung kleinräumiger Wasserversorgungsnetze besteht ein Modellvertrag, der zwischen der Gemeinde, der Verbrauchervereinigung und dem beauftragten Unternehmen - ein Händler, eine Genossenschaft, eine Einzelfirma, eine Gesellschaft mit beschränkter Haftung $(\mathrm{GmbH})$ oder eine Aktiengesellschaft (AG) - abgeschlossen wird. Das Spektrum der Versorger ist äusserst breit. Selbstverständlich setzt der Abschluss eines Vertrags voraus, dass zwischen der Gebietskörperschaft und der Verbrauchervereinigung eine entsprechende Vereinbarung getroffen wurde. Der Vertrag legt fest, unter welchen Bedingungen gewisse spezifische Kompetenzen im Bereich der Wasserversorgung übertragen werden. Die Konzession für das Mandat wird vom zuständigen Ministerium per Beschluss für eine Dauer von drei Jahren vergeben. Die Pflichten der an der Bewirtschaftung der Wasserversorgung beteiligten Akteure sind vertraglich festgelegt.

Der Beauftragte ist dabei die zentrale Figur, denn er verkörpert den Privatsektor. Seine Aufgabe ist es, durch den Einsatz der ihm anvertrauten Anlagen die Wasserversorgung zur Zufriedenheit der Verbraucher sicherzustellen. Dazu zählen der Unterhalt der Bohrung und der übrigen Infrastrukturen und Anlagen, die Beförderung des Trinkwassers bis zum Verbraucher sowie die Pflege der Beziehungen mit den Konsumentinnen und Konsumenten. Er trägt die Betriebskosten, entrichtet der Verbrauchervereinigung regelmässig die vereinbarte Gebühr und nimmt alle übrigen vertraglich vereinbarten Pflichten wahr.

Auf der anderen Seite ist es die Aufgabe der Verbrauchervereinigung, die Funktionstüchtigkeit der Anlagen sicherzustellen, die ihr von der Gebietskörperschaft übertragen wurden. Dazu gewährt sie dem Beauftragten die Unterstützung, die dieser zur Wahrnehmung seiner Pflichten unter den bestmöglichen Bedingungen benötigt. Die Aufgaben der Vereinigung sind die Vertretung gegen aussen, den Schutz der Fassungszonen usw.

42 Siehe dazu das Interview mit Zibo Zakara, früherer Direktor der Wasserversorgung beim Wasser- und Umweltministerium, Sahel Dimanche, 31.12.1998. 
Die Gemeinde, die vom Staat die Eigentumsrechte an der Infrastruktur übernommen hat, muss die Bedürfnisse der Bevölkerung decken, indem sie mit den verschiedenen beteiligten Akteuren zusammenarbeitet und als Aufsichtsbehörde die Versorgungsqualität überwacht.

Die Aufgaben des Staates beschränken sich nunmehr auf verschiedene Unterstützungsfunktionen im Zusammenhang mit der Archivierung und der Prüfung der Geschäftsberichte sowie auf die Bereitstellung technischer Informationen über die Wasserressourcen. Diese Aufgaben werden von den dezentralisierten Diensten des zuständigen Ministeriums wahrgenommen. Damit spielt der Staat eine unterstützende und beratende Rolle. Bei Bedarf beteiligt er sich auch an der Schlichtung von Streitigkeiten im Zusammenhang mit dem Betrieb, die sich im Rahmen des Mandates des Beauftragten ergeben können.

\section{Schema 3: Die ÖPP im Wassersektor des Nigers - Dörfliche Wasserversorgung}

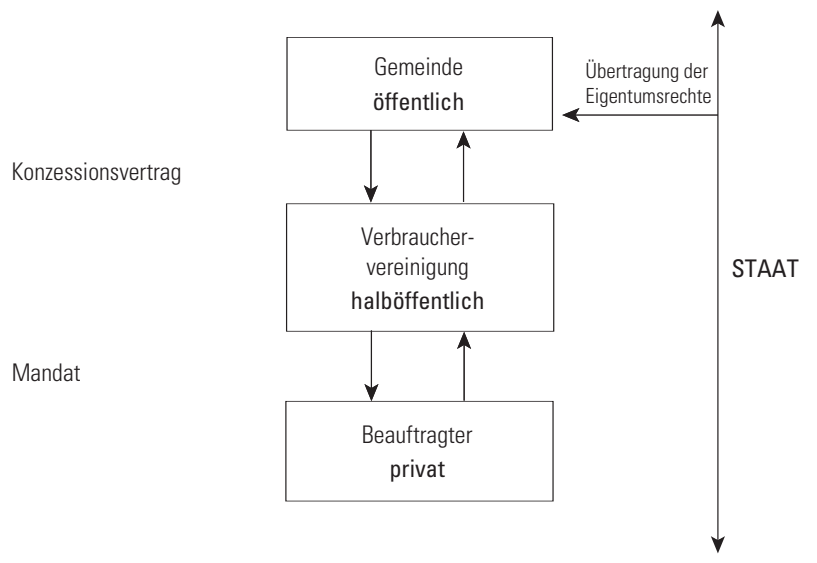

\section{ÖPP im Wassersektor des Nigers: Die Hypotheken}

Die sich institutionalisierende ÖPP im Niger entwickelt vielfältige Erscheinungsformen, welche die unterschiedlichen Strategien und die verschiedenartigen Ausprägungen der veränderten Rolle des Staates verdeutlichen. Dieser steht heute neuen Akteuren gegenüber, die sich selbst noch in einem Lernprozess befinden und gefordert sind, sich in einem neuen institutionellen Kontext zu behaupten.

Im Bereich der urbanen und semiurbanen Wasserversorgung steht die ÖPP im Zeichen der Privatisierung öffentlicher Unternehmen, die von der Weltbank als wichtigster Geldgeberin verfochten wird. Es handelt sich dabei um eine „klassische" Form der ÖPP, in deren Rahmen sich staatliche und private Akteure unter der Aufsicht einer unabhängigen Regulierungsbehörde ergänzen. Entgegen den Befürchtungen, die zu Beginn des Reformprozesses geäussert worden waren namentlich seitens der Gewerkschaften, welche die Privatisierung systematisch mit der Veräusserung von Volkseigentum gleichsetzten ${ }^{43}$-, ging es hier nicht um

43 Siehe dazu das Interview mit dem Generalsekretär der Union des syndicats des travailleurs du Niger, USTN (Dachverband der nigrischen Gewerkschaften), Le Démocrate, 24.2.1997, S. 3. 
den Verkauf eines Staatsbetriebes. Statt dessen wurde die Wasserbewirtschaftung an eine eigens zu diesem Zweck gegründete private Unternehmung verpachtet. Das neue Unternehmen mit Véolia als Mehrheitseigentümerin ist für sämtliche Aktivitäten im Zusammenhang mit der Gewinnung und dem Verkauf von Wasser in urbanen und semiurbanen Zonen zuständig. Damit hat der Staat den hoch profitablen Handel mit Wasser an ein Versorgungsunternehmen von Weltruf abgetreten.

Die Anlagen bleiben zwar im Eigentum des Staates, werden aber auf einen Staatsbetrieb übertragen - die im Zuge der Reform gegründete SPEN -, welcher damit zum wichtigsten Ansprechpartner des neuen Versorgungsunternehmens (SEEN) wird. Darüber hinaus ist die SPEN für die Investitionen der öffentlichen Hand zuständig. In dieser Funktion verwaltet sie die von der SEEN entrichteten Gebühren und pflegt die Beziehungen zu den Geldgebern, die ihre Investitionsbereitschaft von der Umsetzung der Reform abhängig gemacht haben. Damit ist die Société de Patrimoine des Eaux du Niger die wichtigste Ansprechpartnerin des PSE.

Das Beziehungsgeflecht ist in der Tat recht komplex, umfasst es doch neben dem Staat (öffentlich) eine öffentliche Gesellschaft (halböffentlich), ein privatwirtschaftliches Unternehmen (privat) sowie eine unabhängige Instanz (halböffentlich), welche die Einhaltung des neuen gesetzlichen Rahmens überwacht.

In Bezug auf die dörfliche Wasserversorgung deckt das Schema nicht die Gesamtheit der Wasserversorgungsanlagen ab. Es bezieht sich in erster Linie auf die kleinräumigen Wasserversorgungsnetze, welche bislang zumeist gemeinschaftlich bewirtschaftet wurden. In dieser Konstellation sorgt der Staat für die Archivierung von Dokumenten und bietet Unterstützung und Beratung auf verschiedenen Ebenen an. In gewissen Fällen ist er selbst Leistungserbringer, allerdings ist er auf der lokalen Ebene kaum präsent. Als neue Akteure haben sich die 265 Gemeinden etabliert, die im Zuge des Dezentralisierungsprozesses im Niger entstanden $\operatorname{sind}^{44}$. Diese lassen sich durchaus als öffentliche Akteure bezeichnen, vergleichbar mit dem Staat auf nationaler Ebene. Als Eigentümerin der Wasserversorgungsanlagen tritt die Gemeinde deren Bewirtschaftung an die Verbrauchervereinigung ab. Die Verbrauchervereinigungen sind an die Stelle der bis vor Kurzem üblichen Verwaltungsausschüsse getreten und bieten den lokalen Gemeinschaften die Möglichkeit, in einem formellen Rahmen zu handeln. Die Verbrauchervereinigung ihrerseits schliesst mit dem privaten Versorger eine Partnerschaft ab. Das Versorgungsmandat beruht auf einem Pachtvertrag für ein begrenztes Gebiet, welches der Ausdehnung des kleinräumigen Wasserversorgungsnetzes entspricht. Schliesslich sieht der institutionelle Rahmen keine Regulierungsbehörde vor. Ihre Aufgabe wird in gewisser Weise von den dezentralisierten staatlichen Stellen wahrgenommen.

Der Staat ist zwar sowohl in der urbanen, als auch in der dörflichen Wasserversorgung präsent, er nimmt aber je nach Konstellation unterschiedliche Aufgaben wahr. Auch die Rechtsform der privaten Akteure ist in städtischen und ländlichen Gebieten nicht dieselbe. In beiden Fällen aber hat der Staat den grössten

44 Der Dezentralisierungsprozess im Niger wurde mit der Schaffung der Gemeinden im Anschluss an die Wahlen vom 24. Juli 2005 in Gang gesetzt. In dieser neu geschaffenen lokalpolitischen Arena wird sich entscheiden, wie die Gemeinden ihre Rolle in der Wasserversorgung wahrnehmen werden. 
Teil seiner angestammten Zuständigkeiten an neue, sowohl private als auch öffentliche Akteure abgetreten.

Trotz der Vielzahl der beteiligten Parteien erfüllt die Neuordnung des Wassersektors ihren Zweck. Die gegenwärtige Dynamik des Sektors ist zu einem Grossteil darauf zurückzuführen, dass gerade diese Neuordnung den Zugang zu den bedeutenden Finanzmitteln ermöglich, welche die Geldgeber dem Niger zur Verfügung stellen. Auf Grund ihrer strategischen Stellung nehmen die Geldgeber eine zentrale Rolle ein, und sie verfolgen die Entwicklung mit grosser Aufmerksamkeit.

Indessen hängt die Nachhaltigkeit der Reform von der Fähigkeit aller beteiligten Akteure ab, ihren jeweiligen Rollen gerecht zu werden und ihre neuen Aufgaben langfristig wahrzunehmen. Um seine gegenwärtige Stellung zu wahren, muss der Staat sein Know-how in Bezug auf die neu errichteten Bewirtschaftungsformen ausbauen. Die Behörden müssen das rechtliche Instrumentarium beherrschen lernen und ihre Fähigkeiten zur Begleitung des Reformprozesses erweitern. Entsprechend der Auffassung von Ménard ist auf dieser Ebene die Glaubwürdigkeit der konzedierenden Behörde (d.h. des Staates) von zentraler Bedeutung: Er muss gewillt sein, die Reform zu Ende zu führen und namentlich darauf achten, dass dem Ermessensspielraum - namentlich seitens der Behörden - Grenzen gesetzt werden ${ }^{45}$.

Die Société de Patrimoine des Eaux du Niger ihrerseits wird den Beweis für ihre Fähigkeit zur effizienten Bewirtschaftung der ihr anvertrauten Güter erbringen müssen. Sie muss ihr finanzielles Gleichgewicht wahren und ohne Finanzspritzen seitens des Staates und der übrigen Geldgeber auskommen. Längerfristig muss sie den Betrieb ausschliesslich über die vom Versorgungsunternehmen entrichteten Gebühren finanzieren können. Der Wasserpreis spielt dabei eine entscheidende Rolle, denn seine Höhe bestimmt den Umfang der finanziellen Ressourcen der SEEN. Die SPEN steht somit vor der Herausforderung, neue Ressourcen $\mathrm{zu}$ erschliessen und zu bewirtschaften und durch weitere Investitionen ihr Wasserversorgungsnetz auszubauen. Ihre Fähigkeit, diese Aufgabe zu bewältigen - die ihr im Rahmen der Reform ausdrücklich zugewiesen wurde wird über die langfristige Tragfähigkeit des neuen Dispositivs entscheiden.

Als Tochter eines bekannten internationalen Konzerns schlägt der Société des Eaux du Niger grosses Misstrauen entgegen - ob zu Recht oder zu Unrecht, sei dahingestellt. Sie muss die gegen sie erhobenen Vorwürfe entkräften und zeigen, dass sie eine rentable Wasserbewirtschaftung sicherstellen kann, damit ein Fiasko wie jenes, das zum Zusammenbruch der SNE geführt hat, künftig vermieden wird. Dazu muss sie die Voraussetzungen für eine optimale Unternehmensführung schaffen (reibungslose Abläufe innerhalb des Unternehmens sicherstellen und gewährleisten, dass der Staat und die anderen Verbraucher ihre Rechnungen bezahlen, usw.) und ihren finanziellen Verpflichtungen gegenüber der Société de Patrimoine des Eaux du Niger nachkommen.

In urbanen Zonen spielt die Autorité de régulation multisectorielle eine strategische Rolle. Sie muss dafür sorgen, dass sämtliche Akteure ihre Pflichten erfüllen, die in der Planungs- und in der Leistungsvereinbarung zum Konzessionsvertrag zwischen der Republik Niger und der SPEN festgehalten sind. Ménard

45 C. Ménard, op. cit., S. 271. 
hat fünf Merkmale identifiziert, die vorhanden sein müssen, damit die Gefahr einer "Geiselnahme“ der Regulierungsbehörde durch den Versorger (SEEN) oder durch die konzedierende Behörde (Staat) eingedämmt werden kann, nämlich die Trennung zwischen Aspekten der Wirtschaftlichkeit und der Gerechtigkeit, die politische und finanzielle Autonomie des Regulierungsorgans, der Stellenwert der fachlichen Kompetenzen bei der Wahl ihrer Mitglieder, die klare Umschreibung ihrer Zuständigkeiten sowie die Transparenz der Entscheidungsprozesse $^{46}$. Da die ARM erst relativ spät errichtet wurde, ist es für eine fundierte Einschätzung ihrer Arbeit noch verfrüht. Indessen deutet einiges darauf hin, dass gewisse Voraussetzungen bereits gegeben sind, die sie vor einseitiger Einflussnahme schützen. Bereits auf den ersten Blick fallen verschieden Merkmale ins Auge, die den Willen der Behörden belegen, die Unabhängigkeit der ARM zu gewährleisten: das bedeutende Gewicht des Fachwissens als Kriterium bei der Auswahl der Mitglieder des Conseil national de régulation und namentlich bei der Rekrutierung der Direktoren der einzelnen Sektoren über eine internationale Agentur, die Bestimmungen der neuen Gesetzgebung über die Unvereinbarkeit von Ämtern, die umfassenden Zuständigkeiten und Verantwortlichkeiten der Direktoren, die unwiderrufliche Ernennung der Mitglieder des Conseil national de régulation und deren Vereidigung, die Aufgabenteilung zwischen den einzelnen Sektoren sowie die Errichtung einer Website mit einem umfangreichen Angebot an interner Dokumentation. In der Tat lässt die Bilanz der bisherigen Tätigkeit der ARM auf eine gewisse Vitalität schliessen: Bestes Beispiel dafür ist ihr Entscheid im Zusammenhang mit den Beschwerden, die im Anschluss an die Finanz- und Betriebsprüfung für die Rechnungsjahre 2001 und 2004 gegen die SEEN erhoben worden waren ${ }^{47}$.

In den ländlichen Gebieten sind die Probleme bei der Bewirtschaftung der Wasserversorgungsanlagen nach wie vor ungelöst. Die Kommerzialisierung des Wassers bringt neue Herausforderungen und neue Akteure hervor. Die kürzlich errichteten Gemeinden sind gefordert, die Trinkwasserversorgung für die Bevölkerung sicherzustellen, während die Verbrauchervereinigungen mit der Aufgabe konfrontiert sind, die von ihnen beauftragten Versorger zu überwachen und die über Gebühren erwirtschafteten Erträge zu verwalten. Auch die Versorger sind aufgerufen, sich zu professionalisieren, denn die meisten unter ihnen verfügen über keinerlei Erfahrung im Bereich der Wasserversorgung. All diese Hypotheken lasten schwer auf der ÖPP, die gegenwärtig im Niger Fuss zu fassen beginnt.

Der 2001 begonnene schrittweise Aufbau der öffentlich-privaten Partnerschaft im nigrischen Wassersektor wird zehn Jahre in Anspruch nehmen. Im Anschluss an die Aufbauphase wird Bilanz gezogen. Erst dann wird sich zeigen, ob dieses Vorhaben erfolgreich war oder nicht. Unbestritten ist jedenfalls, dass die öffentliche Wasserversorgung im Niger grundlegend neu ausgerichtet wurde. Dennoch ist die Zukunft der ÖPP mit zahlreichen Hypotheken belastet. Alle beteiligten Akteure sind nun aufgerufen, diese Hürden gemeinsam zu überwinden und dafür zu sorgen, dass das eigentliche Ziel der Reform - der Zugang zu Wasser für alle - realisiert wird.

46 C. Ménard, op. cit.

47 Siehe „Décision n ${ }^{\circ}$ D- 2004/03-Ea002“, verfügbar auf der Website der ARM, <www.arm-niger.org>. 


\section{Bibliographie}

\section{Werke, Artikel und Dokumente}

Elhadj DAgobi, A., Olivier de SARdan, J.-P., „La gestion communautaire sert-elle l'intérêt public? Le cas de 1'hydraulique villageoise au Niger", Politique africaine, Nr. 80, 2000, S. 153-168.

Freund B., Lootvoet, B., „Où le partenariat public-privé devient l'instrument privilégié du développement économique et local. L'exemple de Durban, Afrique du Sud“, Revue Tiers Monde, Nr. 181, Januar-März 2005.

Hassane, A., Karadj, A., BAlmer, F. (dir.), Bien gérer l'eau du Sahel. Regards sur vingt ans de coopération entre la Suisse et le Niger dans le secteur de l'hydraulique, Berne, Direction du développement et de la coopération (DDC); Niamey, Ministère de l'hydraulique et de l'environnement; Genève, IUED, 1996.

Jobert, B., Muller, P., L'Etat en action. Politiques publiques et corporations, Paris, Presses Universitaires de France, 1987.

LAIMÉ, M., „Main basse sur l'eau des villes“, Le Monde diplomatique, März 2005, S. 16-17.

Le Monde: Dossiers et Documents, „Saga Vivendi Universal : un rêve en échec“, Nr. 320, Mai 2003.

MÉnARD, C., „Enjeux d'eau: la dimension institutionnelle“, Revue Tiers Monde, Nr. 166, April-Juni 2001, S. 261-274.

Ministère de L'Hydraulique et de L'enVIROnnement, Politiques et stratégies pour l'eau et l'assainissement. De l'eau pour un développement durable, Niamey, Mai 2001.

MinISTÈRE DES FINANCES ET DU PLAN, Assemblée générale des entreprises publiques et parapubliques, rapport général, März 1996.

Plane, P., „La privatisation de l'électricité en Côte d'Ivoire: évaluation et interprétation des premiers résultats“, Revue Tiers Monde, Nr. 152, Oktober-Dezember 1997.

SidiBÉ, S., La réforme du secteur parapublic au Niger, Niamey, Nouvelle Imprimerie du Niger, (ohne Datum).

SociÉTÉ D'Exploitation DEs EAux DU Niger, Assistance du maître d'ouvrage „SPEN“ à la réalisation des bornes fontaines, rapport final, September 2004

\section{Gesetze, Reglemente, Beschlüisse}

„Contrat de concession entre la République du Niger et la Société de Patrimoine des Eaux du Niger (SPEN)“, März 2001 (Konzessionsvertrag zwischen der Republik Niger und der Société de Patrimoine des eaux du Niger SPEN).

„Décision n D- 2004/03-Ea002“, verfügbar auf der Website der ARM, <www.arm-niger.org>.

„Loi 2000-12 du 14 août 2000, portant réorganisation de l'activité de production, transport et distribution de l'eau dans le sous-secteur de l'hydraulique urbaine et créant la Société de Patrimoine des Eaux du Niger (SPEN)“ (Gesetz, über die Reorganisation der Trinkwassergewinnung, -verteilung und -versorgung und über die Gründung der Société de Patrimoine des Eaux du Niger SPEN).

Ministère de L'hydraulique et DE L'Environnement, Programme hydraulique Suisse Niger, Le rôle de l'Etat dans la gestion des ressources naturelles. Recueil des textes législatifs et réglementaires, Niamey, 1999.

Ministère Du PLAN, Ministère DES RESSOURCES EN EAU, Lettre de politique sectorielle de l'hydraulique urbaine, Niamey, 8. März 2001.

„Ordonnance No. 93-014 du 2 mars 1993 portant régime de l'eau“ (Verordnung über die Wasserbewirtschaftung), revidiert durch das Gesetz Nr. 98-041 vom 7. Dezember 1998.

„Ordonnance No. 96-062 du 22 octobre 1996“.

„Ordonnance No. 99-044 du 26 octobre 1999 portant création, organisation et fonctionnement d'une autorité de régulation multisectorielle" (Verordnung über die Errichtung, Organisation und Arbeitsweise einer multisektoriellen Regulierungsbehörde).

\section{Internet-Adresse}

Autorité de régulation multisectorielle (ARM) : <www.arm-niger.org $>$.

\section{Presse}

Alternative Magazine

Le Démocrate

Le Régulateur

Le Républicain

Sahel Dimanche 